\title{
Tungsten coatings under high thermal loads in JET and Magnum-PSI
}

\author{
C Ruset $^{1}$, H Maier ${ }^{2}$, E Grigore ${ }^{\mathrm{a}}$, G F Matthews ${ }^{3}$ G De Temmerman ${ }^{4}$, A Widdowson ${ }^{3}$ \\ and JET-EFDA contributors ${ }^{5}$ \\ JET-EFDA Culham Science Centre, Abingdon, OX14 3DB, UK. \\ ${ }^{1}$ National Inst. for Laser, Plasma and Radiation Physics, Association Euratom-MEdC, Bucharest, \\ Romania \\ ${ }^{2}$ Max-Planck-Institut für Plasmaphysik, EURATOM Association, 85748 Garching, Germany \\ ${ }^{3}$ Euratom/UKAEA Fusion Association, Culham Science Centre, Abingdon, UK \\ ${ }^{4}$ Dutch Institute for Fundamental Energy Research, Association EURATOM-FOM, Netherlands \\ ${ }^{5}$ See the Appendix of F. Romanelli et al., Proc. of the 24th IAEA Fusion Energy Conference, 2012, San \\ Diego, US
}

\begin{abstract}
During the C28-C30 campaigns the ITER-like Wall (ILW) was periodically inspected by IVIS (In Vessel Inspection System) without breaking the vacuum. After these campaigns the JET chamber was opened and the wall was inspected with a high resolution camera. The marker tiles were removed from the chamber and they have been inspected in the beryllium handling facility. The overall picture of the ILW after more than 3000 pulses looks good. However there are a few modifications such as deposition of Be on some W coated tiles, local destruction of the W coating by runway electrons in an emergency shutdown, arcing on particular $\mathrm{W}$ coated tiles, small delaminations on G6 and G8 divertor tiles. These aspects together with the results of high heat flux tests carried out with W coated CFC samples in Magnum-PSI are discussed in the paper.
\end{abstract}

Corresponding author: Cristian Ruset, E-mail: ruset@infin.ro 


\section{Introduction}

During the last three campaigns (August 2011-July 2012) JET has been operated with the new ITER like wall (ILW) containing bulk Be tiles (main chamber), bulk W lamellas (divertor G5 tiles) and W coated CFC tiles. The thickness of the coating is $10-15 \mu \mathrm{m}$ for the main chamber neutral beam shinethrough related tiles plus some critical recessed areas of the wall and divertor tiles G3, G4. Thicker 20-25 $\mu \mathrm{m}$ W coatings were used for divertor tiles G1, G6, G7 and G8. All W coatings included a Mo interlayer of 2-3 $\mu \mathrm{m}$ between $\mathrm{W}$ layer and CFC substrate. A picture with JET divertor area and tiles identification is shown in Fig. 1. In the present configuration the ILW contains about 1300 tungsten coated CFC tiles. All the W coatings were produced by Combined Magnetron Sputtering and Ion Implantation (CMSII) technology which involves periodical high energy ion bombardment of the coating during its growth [1]. During JET operation W coatings are subjected to quasi-stationary thermal loading $(10-20 \mathrm{~s})$ and transients due to ELMs ( $\tau=1-2 \mathrm{~ms}, \mathrm{f} \sim 10-20 \mathrm{~Hz}$ ). As far as long pulse thermal loading is concerned high heat flux (HHF) tests have been carried out on W coatings in the GLADIS hydrogen beam facility (IPP Garching) during the qualification and production phases of the ILW project [2]. Additional tests have been performed with the electron beam high temperature test facility at NILPRP, Bucharest with the aim to investigate the limits of these $\mathrm{W}$ coatings for a large number of heating cycles [3]. ELM-like simulations tests with duration of $\sim 1$ ms were carried out on the same type of W coatings in JUDITH 1 and JUDITH 2 facilities at FZ Jülich [4]. Recently the W coatings were tested in Magnum-PSI [5] (DIFFER, Nieuweigein, Netherlands) which can apply simultaneous steady state plasma fluxes and ELMlike transients. The results are presented in this paper. All these tests provided information about the capability of the W coatings produced by CMSII technology to sustain high thermal loads in different testing conditions. They have been performed with the aim of anticipating the behavior of $\mathrm{W}$ coatings in JET to provide a basis for defining allowable operating limits for the coatings. Local coating failures may be responsible for generation of $\mathrm{W}$ particles leading to transient radiation events [6] and if sufficient areas were affected could also lead to a rise in carbon levels which would impact fuel retention and plasma behavior [7]. During the JET ILW campaigns the inner strike point was always operated on the tungsten coatings but the outer strike point was mostly positioned on the bulk tungsten tile. In future campaigns, more outer strike point operation on tungsten coatings is planned [8].

\section{Testing of $W$ coatings in Magnum PSI}

A CFC sample with approximate dimensions $80 \times 70 \times 40 \mathrm{~mm}^{3}$ has been coated with $\mathrm{W}$ layer of 20 $\mu \mathrm{m}$ perpendicular to fiber planes using CMSII technology. The sample was installed in Magnum-PSI on water cooled plate and it was tested at the following parameters: (i) Plasma source power: about $40 \mathrm{~kW}$; (ii) Working gas: hydrogen; (iii) Magnetic field: $1.3 \mathrm{~T}$; (iv) Shot duration: $12 \mathrm{~s}$; (v) Pulse duration: about $1 \mathrm{~ms}$; (vi) Pulse frequency: 5-20 Hz; (vii) Pulse energy transferred to the sample was in the range of 55-85 J; (viii) Total number of shots: 30; (ix) Total number of pulses: 2875.

The plasma beam with a diameter of about $30 \mathrm{~mm}$ was centered on the area $80 \times 70 \mathrm{~mm}$ of the sample. The temperature was measured with an IR camera at a frequency of $4 \mathrm{kHz}$ on the wavelength of 4 $\mu \mathrm{m}$. Under these conditions the base temperature due to the steady state plasma started at $\sim 40{ }^{\circ} \mathrm{C}$ and reached after $12 \mathrm{~s}$ a temperature of $\sim 315^{\circ} \mathrm{C}$. The base temperature at which thermo-shocks were applied was $\sim 200-315{ }^{\circ} \mathrm{C}$. The increase of surface temperature due to short pulses was about $100-120{ }^{\circ} \mathrm{C}$. The time dependence of the $\mathrm{W}$ coating temperature is shown in Fig.2.

The coating was examined with a stereomicroscope (magnification 40x) after 1675 and 2875 pulses. No delaminations or other damages have been detected except two small spots with the dimensions of 100-200 $\mu \mathrm{m}$ where the $\mathrm{W}$ coating was melted, but without detachment from the substrate. The melting was probably due to some arcs. The estimated power density of the ELM-like pulses was $80-120 \mathrm{MW} / \mathrm{m}^{2}$ which is above the values calculated for JET with the ILW [9]. The steady state power density during the experiments was about $10 \mathrm{MW} / \mathrm{m}^{2}$.

\section{Inspection of the $W$ coatings from the ILW}


The status of the ILW was periodically monitored during the C28-C30 campaigns using IVIS (In Vessel Inspection System) without breaking the vacuum. Four special cameras were available to inspect the entire wall from the top to divertor area. Although the resolution of the cameras was not very high, these inspections provided useful information about the status of the tiles at different moments in C28-C30 campaigns.

After C30 campaign when the JET vessel was opened a high performance camera was introduced into the vessel by remote control system and detailed pictures were taken for all components of the ILW.

A set of seven W/Mo/W/Mo marker tiles were withdrawn from the vessel with the aim to be analysed after one year of JET exploitation and on this occasion close up pictures were taken in the beryllium handling facility (BeHF).

From the HHF tests carried out outside of JET we have learned that the damage of the W coatings could be classified in two categories: microscopic and macroscopic.

The microscopic damage consisted mainly of buckling with dimensions of 30-500 $\mu \mathrm{m}$ [10]. In this case on small areas the coating has lost the contact with the CFC substrate, but it was not removed. If the power density is not high enough to melt the thin $\mathrm{W}$ layer, the chip remains on the surface still playing its role of preventing the plasma-carbon contact. Unfortunately, this type of microscopic damage can only be seen with a stereomicroscope or a scanning electron microscope.

The macroscopic damage consists of delaminations with dimensions higher than $0.5 \mathrm{~mm}$ that usually occur along the in plane fibre of the CFC material. They can be seen with a good digital camera.

It would have been a good idea to inspect the marker tiles from both microscopic and macroscopic point of view, but unfortunately a stereomicroscope was not yet available in the Beryllium Handling Facility (BeHF) where the tiles had to be examined due to deposition of beryllium from the main wall. Consequently, the discussion in this paper will be based only on the information provided by the digital cameras. Small samples cut from the marker tiles will be analyzed microscopically later.

\section{Results and discussion}

The monitoring pulses did not show a substantial increase of the $\mathrm{C}$ level during plasma operation which suggested a good condition of the $\mathrm{W}$ coatings on CFC tiles. Then, the visual inspection confirmed that the overall picture on the W coatings from the ILW looks good. However, some local modifications occurred and the discussion in the paper will be focused on these aspects.

In the main chamber $\mathrm{W}$ coated CFC tiles were introduced in areas with the highest thermal loading. In the Inner Wall Guard Limiter (IWGL) they are near to the Be tiles. The pictures of those areas show relative thick deposits of material (probably Be) on the W coated CFC tiles ("wings"). This can be seen in Fig.3 taken in Octant 7, inner wall, bottom of the limiter beam. On the limiter there is a transition from strong erosion (in the centre area) to deposition (in the side zones). The ridge of the Be tiles was eroded or even melted in some cases [11] and material was deposited on the W-coated wing tiles. The same phenomenon has been seen in Octant 3 (inner wall, bottom of the limiter beam), Octant 5 (outer wall, mid-plane), Octant 6 (top limiter beam) and Octant 8 (top limiter beam). This is clear a pattern, specific for this type of wall. The other W coated CFC tiles shown in Fig.3 appear to be in very good condition.

A thorough inspection of the $\mathrm{W}$ coated CFC tiles revealed the damage of one bottom tile, Inner Wall Guard Limiter placed in the same position in Octants 2, 5 and 7. It is shown in Fig.4. The W coating seems to be melted on the left round edge of the tile. The damage seems to be produced by the runway electrons in the early commissioning phase of the ILW during an emergency shutdown (pulse 80263).

Another phenomenon which was observed after the first campaigns is arcing on some particular tiles. These are located on outer wall, shine through protection, Octants 1 and 5. An image is shown in Fig.5. The pattern of the damages is the same in both octants. Two of W-coated CFC tiles from Octant 1 were removed and inspected carefully with a high resolution camera in the BeHF. The following aspects were revealed:

- The W coating was subjected to intense arcing. This leads to delamination of the coating along the arc track and even melting of the $\mathrm{W}$ coating. This is evidenced in Fig.5 (inset). 
- In local zones the temperature during arcing was so high that the CFC substrate was affected. Black spots surround the $\mathrm{W}$ melted zones indicating that carbon was eroded from the substrate and deposited onto the $\mathrm{W}$ coating.

A clear explanation on the arcing phenomenon on those particular tiles was not found yet. It might be associated with the presence of some small carbon particles resulting from mechanical processing of the CFC tile after $\mathrm{W}$ coating or related to the earthing of particular tiles to the vessel leading to local variations in potential during extreme events such as disruptions. On the other hand some tiles were hand cut after W coating to correct clashes discovered during the installation of the ILW without arc signs. More investigations are necessary in order to clarify the presence of such intense arcs on these tiles.

As far as the $\mathrm{W}$ coatings deposited on divertor tiles are concerned their current status is generally good except minor delaminations detected on a G6 tile, which are probably associated with the CFC structure and some delaminations on the top of about twenty G8 tiles, which seems to be systematic. All delaminations with a length of 1-15 mm are along the in plane fibers of the CFC material (Fig.6). The W coating is perfect on the main active area of the G8 tiles, where the thermal loading is supposed to be high, but the defects appeared only on the top of the tiles. The first signs of delamination appeared on the top of G8 in October 2011 after about two months of experiments. Examination of the pictures taken with IVIS on the same G8 tiles in Octant 8 in the period October 2011-May 2012 revealed no increase of the damages. The inspections revealed the presence of many small particles dropped on this horizontal surface of G8 tiles. Without any contact with the substrate these particles become very hot during plasma operation and in this way the temperature of the $\mathrm{W}$ coating is locally increased to high values. These hot spots might affect the W coating integrity. The strong erosion on the top of G8 tiles was noticed in 2007 with the carbon wall when a marker tile was used [12]. The defects on G6 and G8 appear random in that some tiles show a significant number while adjoining tiles may show none at all. This strongly suggests that plasma parameters are not the dominant factor and that variations in the CFC substrate are the dominant cause.

\section{Conclusions}

The W coatings of $20 \mu \mathrm{m}$ deposited by CMSII technology on CFC Dunlop DMS 780 material survived the ELM like tests carried out in Magnum-PSI, but the base temperature produced by the steady state plasma was relatively low $\left(\sim 400^{\circ} \mathrm{C}\right)$ in comparison with that obtained by hydrogen beam or with the electron beam which was over $1000{ }^{\circ} \mathrm{C}$. As the power density of the applied ELM-like pulses was higher than that estimated for JET [9] it can be concluded that ELMs are consequently no threat to JET operation.

General behaviour of the W coatings in the ILW during the C28-C30 campaigns (> 3000 pulses) is very positive. Some coating damage has been observed. For example, in the main chamber runaway electrons melted the coating on the bottom tiles of the inner wall guard limiter and there are also signs of arc damage on some tiles. In the divertor, some delaminations are observed on the in-plane fibres of specific tiles but this seems related to the substrate and not the coating process or plasma parameters. Although the focus of this paper is on the damage that has been observed the total area of exposed carbon is extremely small and it is not a significant contributor to the residual carbon levels.

\section{Acknowledgments}

The work was supported by the European Commission and the Romanian Ministry for Education and Research under EURATOM Program EFDA Task Agreement Code: Fusion Technology JW13-FT-4.31 and by LAPLAS Program Code:PN09.39.03.01.

The views and opinions expressed herein do not necessarily reflect those of the European Commission.

\section{References}

[1] C. Ruset, et al., Fusion Engineering and Design 84 (2009) 1662-1665

[2] H. Maier, et al., Phys. Scr. T138 (2009) 014031 (5pp)

[3] C. Ruset, et al., Fusion Engineering and Design, (2013) (in press)

[4] C. Thomser, et al., Fusion Science and Technology, Vol. 62, 1, (2012), Pages:1-8 
[5] G. De Temmerman, et al., Appl. Phys. Lett. 97, 081502 (2010)

[6] M. Sertoli, et al., this conference

[7] G. F. Matthews et al., 20th Int. Conf. on Plasma Surface Interactions in Controlled Fusion, Aachen, 2012, http://dx.doi.org/10.1016/j.jnucmat.2013.01.282)

[8] G.F.Matthews et al., this conference

[9] T. Eich, et al., this conference

[10] H. Maier et al., J. Nucl. Mater 363-365 (2007), 1246

[11] A. Widdowson, et al., this conference

[12] M. Mayer, et al., Journal of Nuclear Materials 363-365 (2007), 101-106

\section{Figure caption}

Fig.1 Cross sectional view of the JET divertor area including tiles identification

Fig.2 Typical temperature time trace of applied thermal shock loads at $5 \mathrm{~Hz}$ in Magnum PSI, super positioned with a steady state heat load of $10 \mathrm{MW} / \mathrm{m}^{2}$

Fig.3 Deposits of Be on W-coated CFC wing tiles which protect the edge of some inner wall limiters from first pass neutral beam shine-through.

Fig.4 Local damage to $\mathrm{W}$ coatings at the bottom of the inner wall limiters which is due to the impact of runaway electrons

Fig.5 Arcing on $\mathrm{W}$ coated shine-through protection tiles designed to protect outer beryllium limiters from tangential neutral beams which pass twice through the plasma

Fig.6 Small delaminations on the horizontal area of G8 tile; the picture was taken in the Be handling facility after removing of the tile from JET chamber 


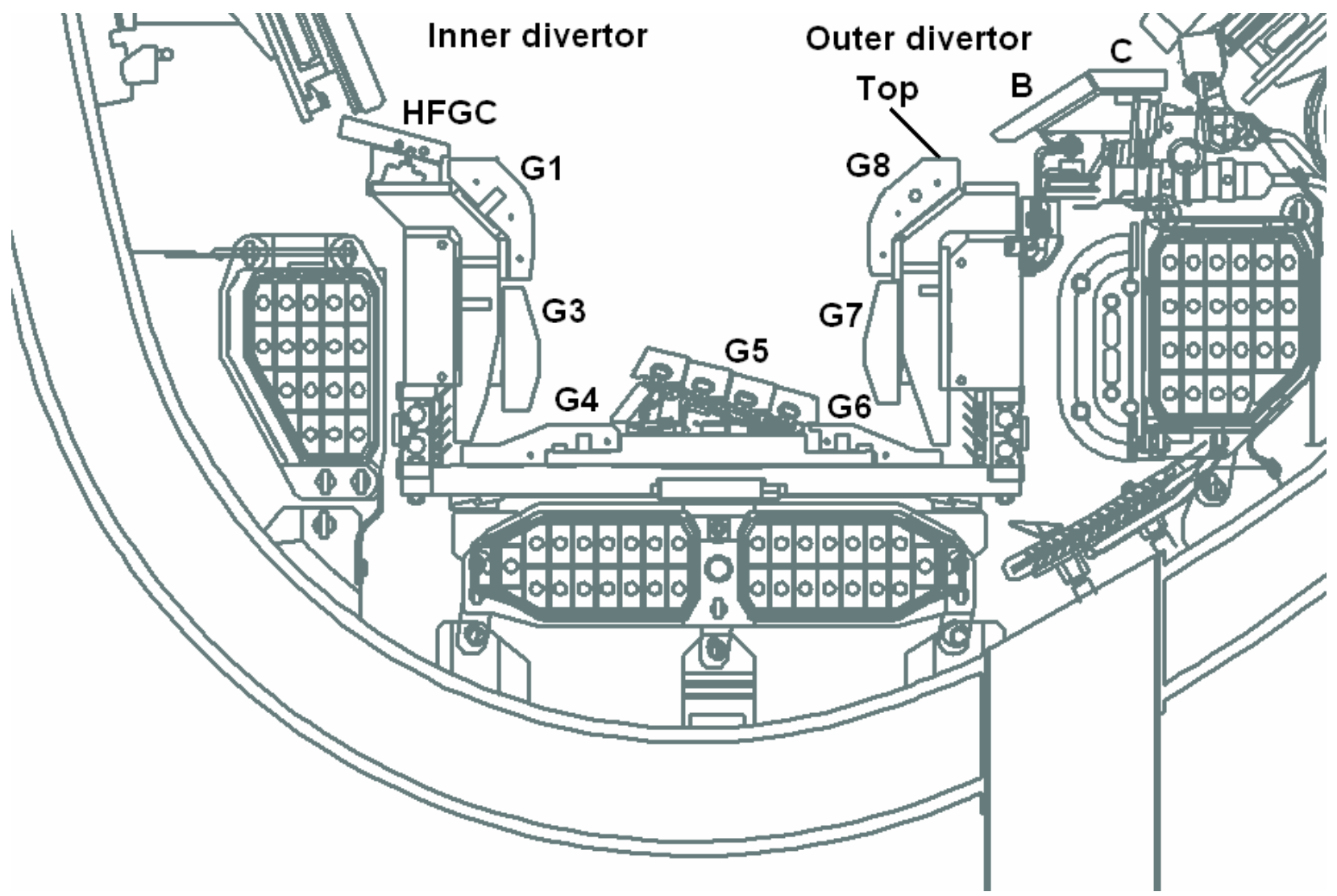

Fig. 1 Cross sectional view of the JET divertor area including tiles identification 
Shot 2, 22/11/12: 260J, $5 \mathrm{~Hz}$ Plasma pulses

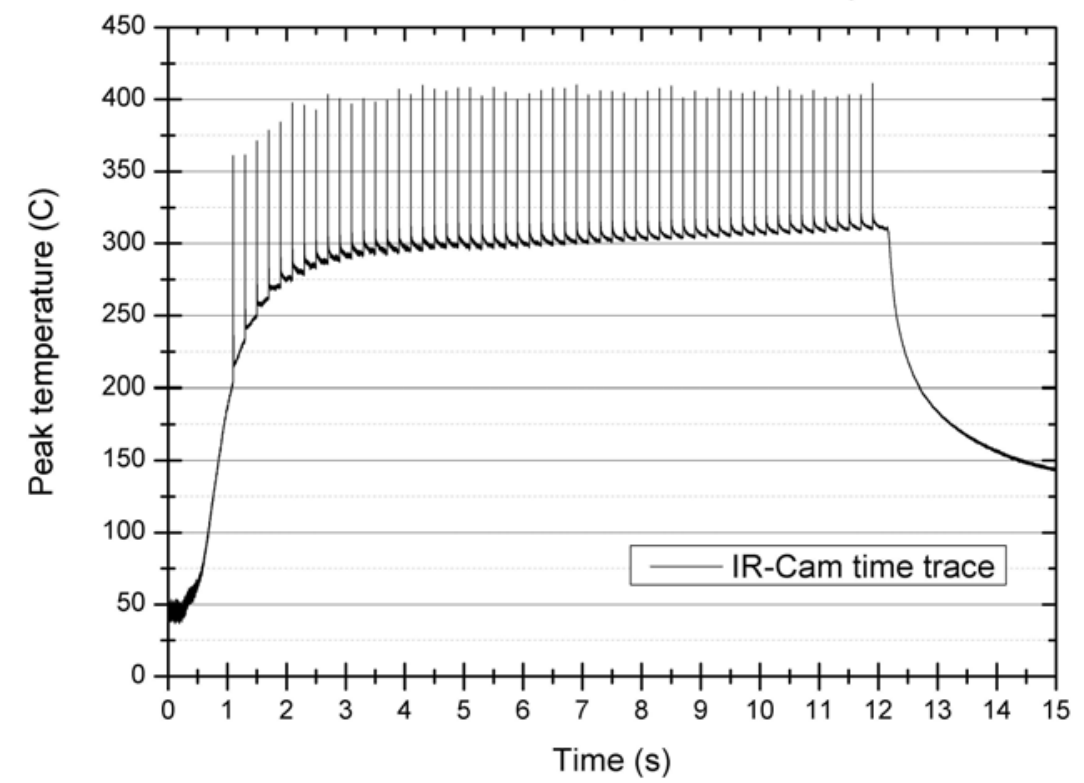

Fig.2 Typical temperature time trace of applied thermal shock loads at $5 \mathrm{~Hz}$ in Magnum PSI, super positioned with a steady state heat load of $10 \mathrm{MW} / \mathrm{m}^{2}$ 


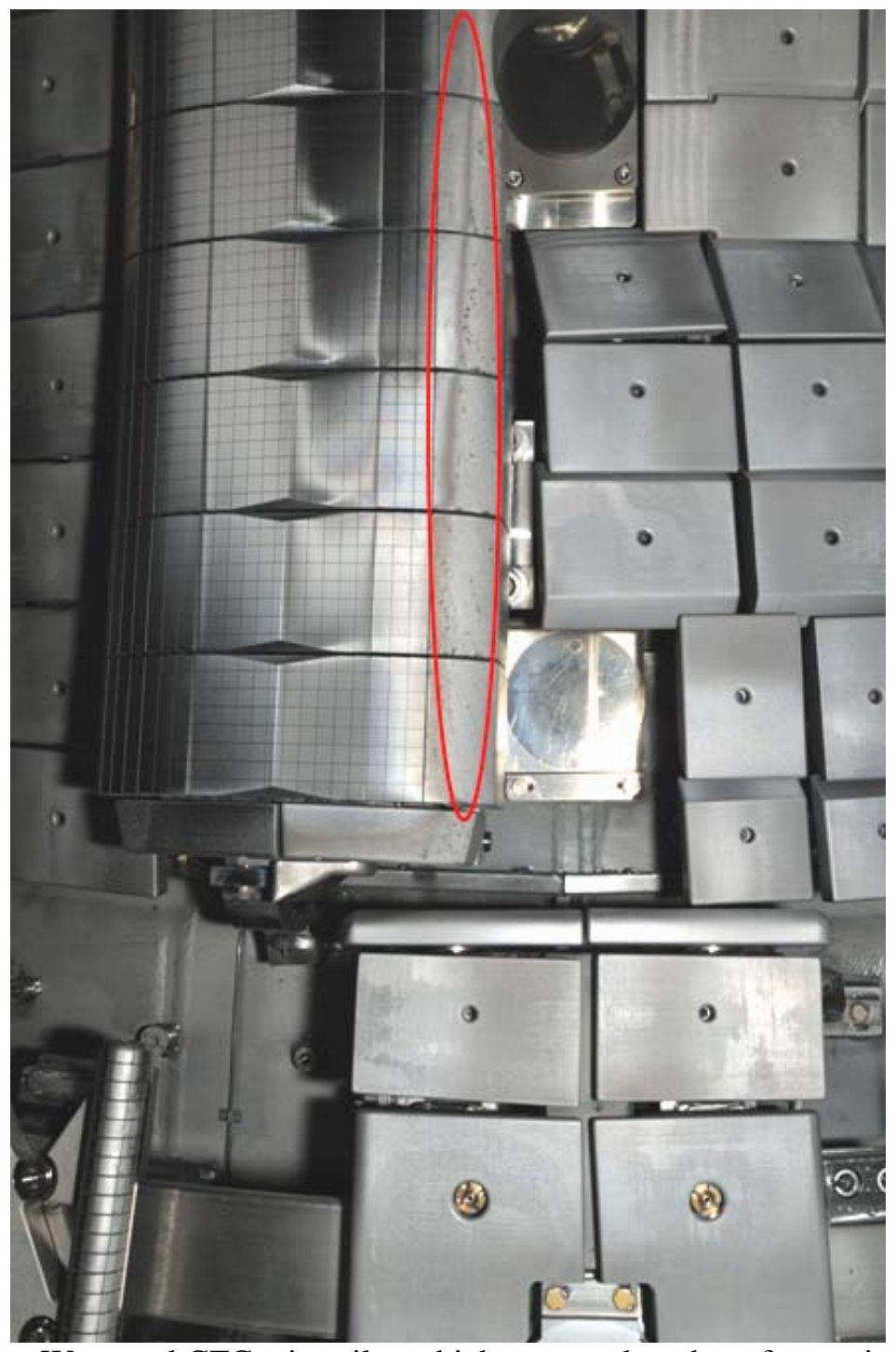

Fig.3 Deposits of Be on W-coated CFC wing tiles which protect the edge of some inner wall limiters from first pass neutral beam shine-through. 


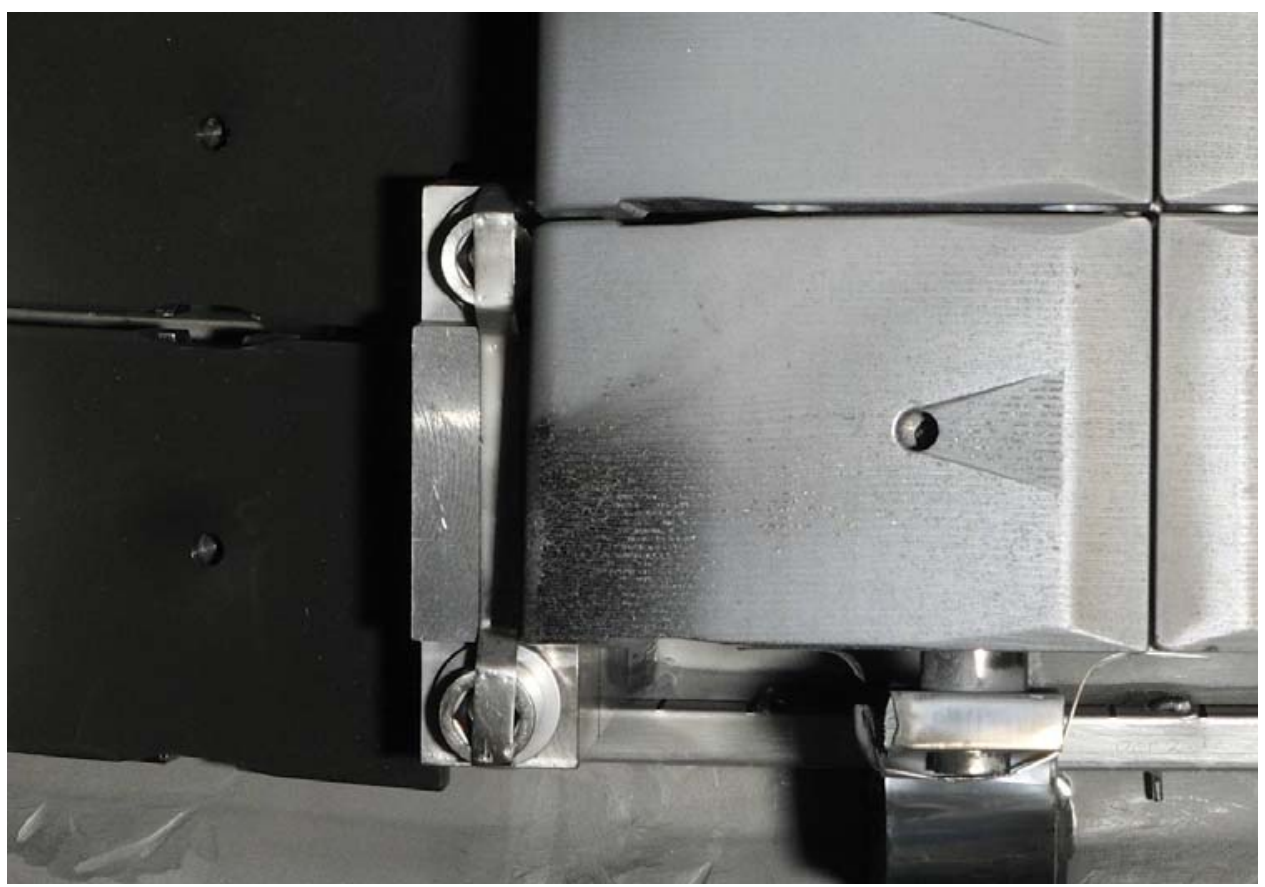

Fig.4 Local damage to $\mathrm{W}$ coatings at the bottom of the inner wall limiters which is due to the impact of runaway electrons 


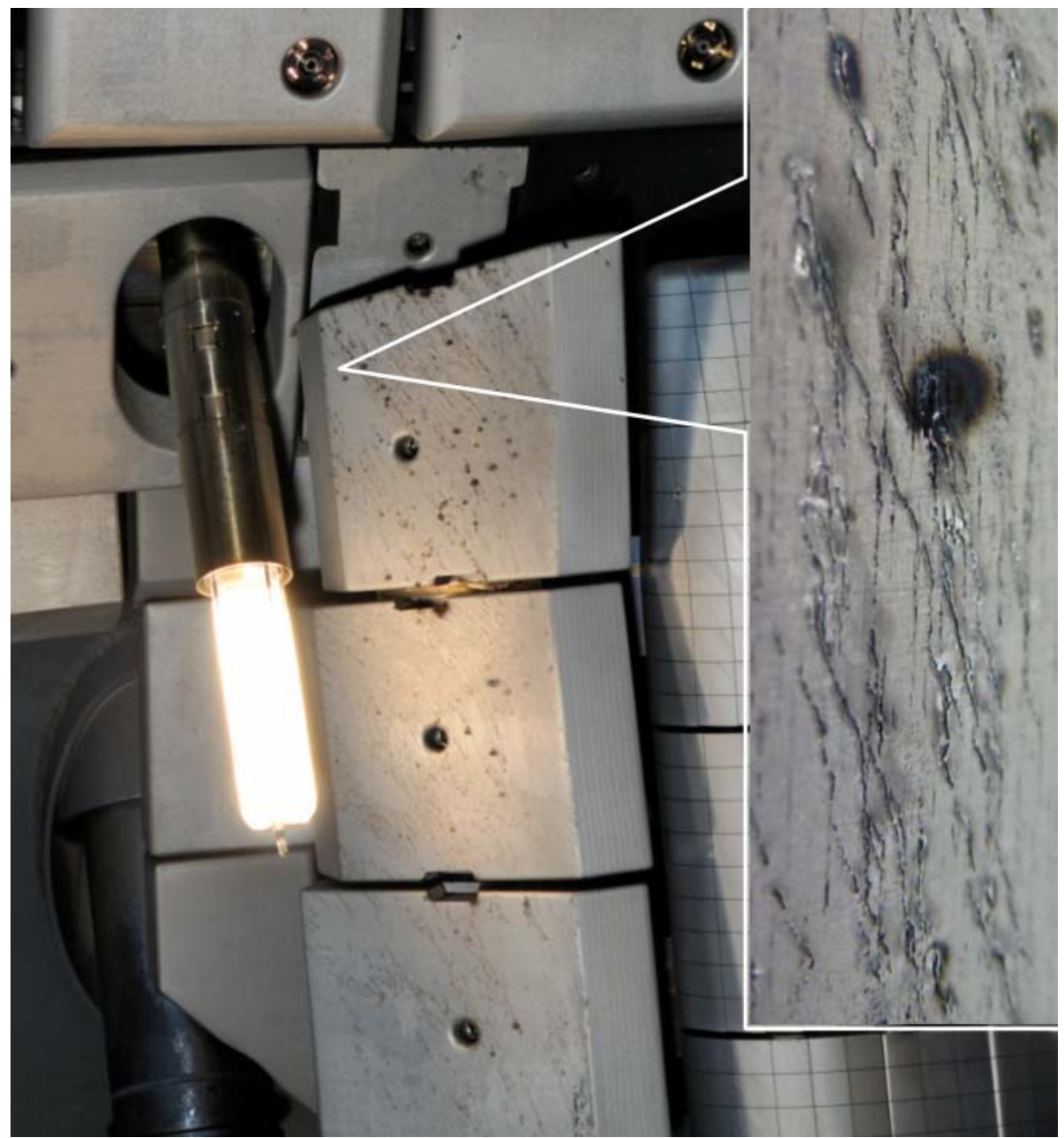

Fig. 5 Arcing on W coated shine-through protection tiles designed to protect outer beryllium limiters from tangential neutral beams which pass twice through the plasma 


\section{Active area}

\section{Horizontal (top) surface}

\section{Small delaminations}

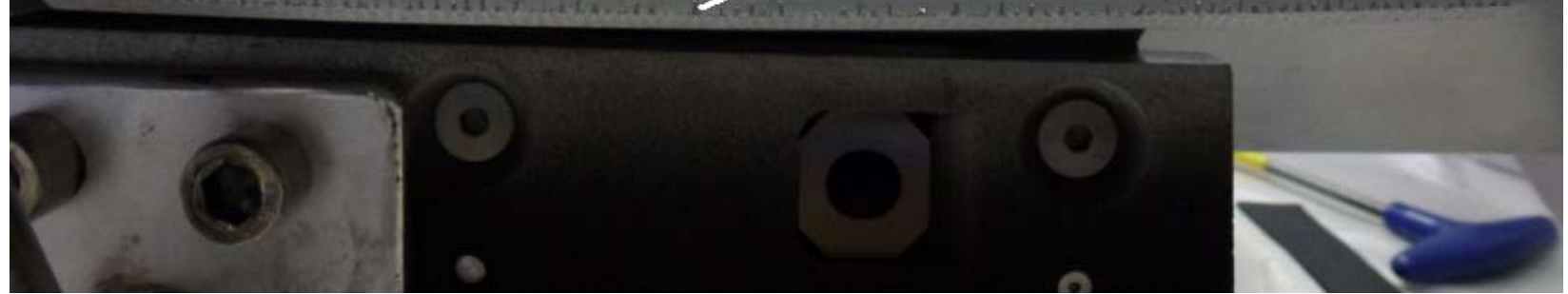

Fig.6 Small delaminations on the horizontal area of G8 tile; the picture was taken in the Be handling facility after removing of the tile from JET chamber 